\title{
First case of COVID-19 complicated with fulminant myocarditis: a case report and insights
}

\author{
Jia-Hui Zeng ${ }^{1}$ - Ying-Xia Liu ${ }^{2}$ Jing Yuan ${ }^{3} \cdot$ Fu-Xiang Wang ${ }^{3} \cdot$ Wei-Bo Wu ${ }^{3} \cdot$ Jin-Xiu Li Li-Fei Wang $^{5} \cdot \mathrm{Hong} \mathrm{Gao}^{6}$. \\ Yao Wang ${ }^{1} \cdot$ Chang-Feng Dong ${ }^{1} \cdot$ Yi-Jun $\mathrm{Li}^{1} \cdot$ Xiao-Juan Xie ${ }^{1} \cdot$ Cheng Feng $^{1} \cdot$ Lei Liu $^{2}$
}

Received: 6 February 2020 / Accepted: 5 April 2020 / Published online: 10 April 2020

(c) Springer-Verlag GmbH Germany, part of Springer Nature 2020

\begin{abstract}
Background Coronavirus disease 2019 (COVID-19) has been demonstrated to be the cause of pneumonia. Nevertheless, it has not been reported as the cause of acute myocarditis or fulminant myocarditis.

Case presentation A 63-year-old male was admitted with pneumonia and cardiac symptoms. He was genetically confirmed as having COVID-19 according to sputum testing on the day of admission. He also had elevated troponin I (Trop I) level (up to $11.37 \mathrm{~g} / \mathrm{L}$ ) and diffuse myocardial dyskinesia along with a decreased left ventricular ejection fraction (LVEF) on echocardiography. The highest level of interleukin- 6 was $272.40 \mathrm{pg} / \mathrm{ml}$. Bedside chest radiographs showed typical groundglass changes indicative of viral pneumonia. Laboratory test results for viruses that cause myocarditis were all negative. The patient conformed to the diagnostic criteria of the Chinese expert consensus statement for fulminant myocarditis. After receiving antiviral therapy and mechanical life support, Trop I was reduced to $0.10 \mathrm{~g} / \mathrm{L}$, and interleukin- 6 was reduced to $7.63 \mathrm{pg} / \mathrm{mL}$. Moreover, the LVEF of the patient gradually recovered to $68 \%$. The patient died of aggravation of secondary infection on the 33rd day of hospitalization.

Conclusion COVID-19 patients may develop severe cardiac complications such as myocarditis and heart failure. This is the first report of COVID-19 complicated with fulminant myocarditis. The mechanism of cardiac pathology caused by COVID19 needs further study.
\end{abstract}

Keywords COVID-19 · Coronavirus · Fulminant myocarditis $\cdot$ Infection $\cdot$ Echocardiography

Cheng Feng

chaosheng-01@szsy.sustech.edu.cn

$\triangle$ Lei Liu

liulei3322@aliyun.com

1 Department of Medical Ultrasonics, National Clinical Research Center for Infectious Disease, State Key Discipline of Infectious Disease, Shenzhen Third People's Hospital, Second Hospital Affiliated to Southern University of Science and Technology, No. 29 Bulan Road, Shenzhen 518112, People's Republic of China

2 National Clinical Research Center for Infectious Disease, State Key Discipline of Infectious Disease, Shenzhen Third People's Hospital, Second Hospital Affiliated to Southern University of Science and Technology, Shenzhen, China

3 Department of Infectious Disease, National Clinical Research Center for Infectious Disease, State Key Discipline of Infectious Disease, Shenzhen Third People's Hospital, Second Hospital Affiliated to Southern University of Science and Technology, Shenzhen, China
4 Department of Intensive Care Unit, National Clinical Research Center for Infectious Disease, State Key Discipline of Infectious Disease, Shenzhen Third People's Hospital, Second Hospital Affiliated to Southern University of Science and Technology, Shenzhen, China

5 Department of Radiology, National Clinical Research Center for Infectious Disease, State Key Discipline of Infectious Disease, Shenzhen Third People's Hospital, Second Hospital Affiliated to Southern University of Science and Technology, Shenzhen, China

6 Department of Cardiology, National Clinical Research Center for Infectious Disease, State Key Discipline of Infectious Disease, Shenzhen Third People's Hospital, Second Hospital Affiliated to Southern University of Science and Technology, Shenzhen, China 


\section{Background}

A series of unexplained pneumonia cases were reported in Wuhan, Hubei Province, China in December 2019, and the clinical manifestations were very similar to viral pneumonia. A new coronavirus was identified and named Coronavirus disease 2019 (COVID-19) after sequencing analyses of samples from patients' respiratory tracts. Common symptoms are fever, cough, myalgia, and/or fatigue. All patients had pneumonia, and chest computed tomography (CT) scans showed abnormalities. Complications include acute respiratory distress syndrome (ARDS), acute cardiac injury, and secondary infection $[1,2]$. Here, we describe a case of COVID-19 complicated with fulminant myocarditis.

\section{Case report}

A 63-year-old male was admitted to the hospital due to the expectoration of white sticky sputum, a fever of $39.3{ }^{\circ} \mathrm{C}$, and shortness of breath and chest tightness after activity. He had no history of heart disease or hypertension. He had allergic cough for 5 years and a previous smoking history. He had a recent history of travel to Hubei Province, China.

Blood gas analysis after admission showed a $\mathrm{pH}$ of 7.049, $\mathrm{PCO}_{2}$ of $76.4 \mathrm{mmHg}, \mathrm{PO}_{2}$ of $66.2 \mathrm{mmHg}$, and $\mathrm{SaO}_{2}$ at $91.8 \%$. Nasal and pharyngeal swabs, bronchoalveolar lavage fluid (BALF), and sputum were tested for common respiratory viruses, and the results were all negative. In addition, blood samples showed increased alanine aminotransferase (ALT) $(97 \mathrm{U} / \mathrm{L})$ and creatinine $(157 \mu \mathrm{mol} / \mathrm{L})$ combined with hematuria, indicating deranged liver and renal function. Bedside chest radiographs showed typical ground-glass changes indicative of viral pneumonia (Fig. 1). On the first day of admission, he was genetically confirmed to have COVID-19 according to sputum testing, as shown in Table 1.

Markers of myocardial injury included elevated troponin I (Trop I) (11.37 g/L), myoglobin (Myo) $(390.97 \mathrm{ng} / \mathrm{mL})$, and n-terminal brain natriuretic peptide (NTBNP) $(22,600 \mathrm{pg} /$ $\mathrm{mL}$ ) (Fig. 2). An electrocardiogram showed sinus tachycardia and no ST-segment elevation (Fig. 3). Bedside echocardiography showed an enlarged left ventricle $(61 \mathrm{~mm})$,
Table 1 The cycle threshold of respiratory specimens

\begin{tabular}{llll}
\hline Day after admission & Pharyngeal swab & Sputum & BALF \\
\hline 1 & - & 22 & $/$ \\
2 & - & $/$ & $/$ \\
3 & $/$ & $/$ & 24 \\
4 & 30 & 22 & $/$ \\
5 & 20 & $/$ & $/$ \\
6 & - & $/$ & $/$ \\
7 & $/$ & 29 & $/$ \\
8 & - & - & $/$ \\
9 & 33 & $/$ & $/$ \\
10 & 31 & $/$ & $/$ \\
11 & $/$ & $/$ & 27 \\
12 & 27 & $/$ & 24 \\
13 & $/$ & $/$ & 27 \\
\hline
\end{tabular}

diffuse myocardial dyskinesia along with a low left ventricular ejection fraction (LVEF) (32\%), pulmonary hypertension $(44 \mathrm{mmHg})$, and no decrease in right cardiac function (Figs. 4, 5). No pericardial effusion was observed.

The diagnosis was considered severe pneumonia, ARDS, fulminant myocarditis, and multiple organ dysfunction syndrome (MODS). The treatment regimen was ventilatory support, high-flow oxygen, lopinavir-ritonavir antiviral therapy, interferon $\alpha-1 b$, methylprednisolone, immunoglobulin, piperacillin-tazobactam, and continuous renal replacement therapy (CRRT). Notably, 11 days after admission, the patient's lung lesions continued to progress, and the ventilator was unable to maintain oxygen saturation. The ventricular septum thickened to $14 \mathrm{~mm}$, and interleukin-6 (IL-6) increased to $272.40 \mathrm{pg} / \mathrm{mL}$. Therefore, extracorporeal membrane oxygenation (ECMO) was used to reduce the cardiopulmonary burden. The rotating speed of ECMO was $3500 \mathrm{rpm}$, the blood flow rate was $4.30 \mathrm{~L} / \mathrm{min}$, the airflow rate was $4.00 \mathrm{~L} / \mathrm{min}$, and $\mathrm{FiO}_{2}$ was $100 \%$. At one time, the patient's condition improved. A blood test 3 days later revealed that Trop I was reduced to $0.10 \mathrm{~g} / \mathrm{L}$, NTBNP was $750 \mathrm{pg} / \mathrm{mL}$, and IL-6 was $7.63 \mathrm{pg} / \mathrm{mL}$. After treatment, the LVEF of the patient gradually recovered to $68 \%$, the left ventricle and wall thickness returned to within their normal ranges, and the bedside chest film suggested that the
Fig. 1 a Bedside chest radiograph on the day of admission; b, c computerized tomography on the ninth day showing typical ground-glass changes indicative of viral pneumonia

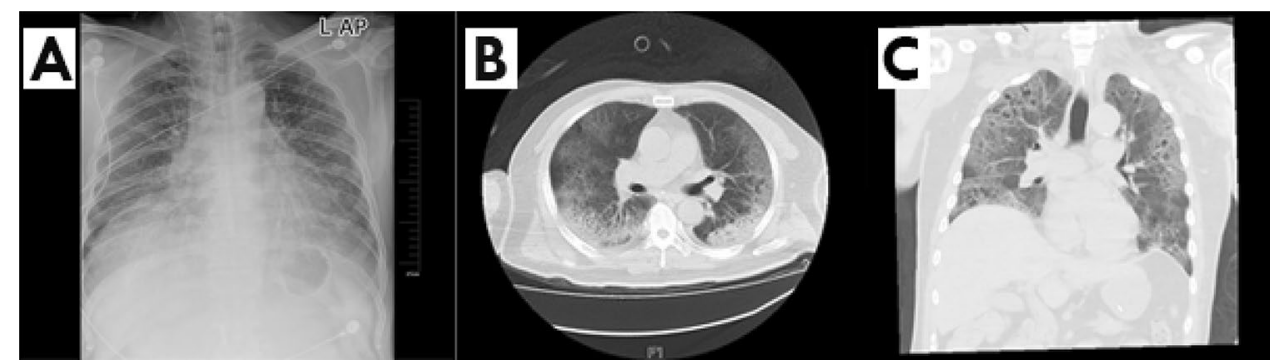


Fig. 2 The line chart of the markers of myocardial injury. Trop I troponin I, MYO myoglobin, NTBNP n-terminal brain natriuretic peptide. Trop I and NTBNP decreased gradually, while no significant decrease in MYO was observed

Fig. 3 The electrocardiogram showing sinus tachycardia and no ST-segment elevation

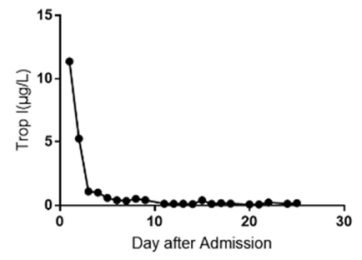

A

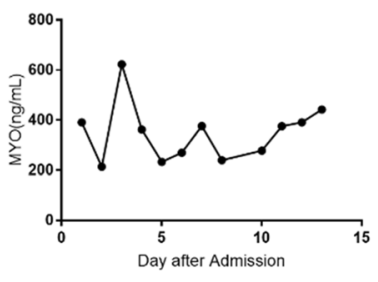

B

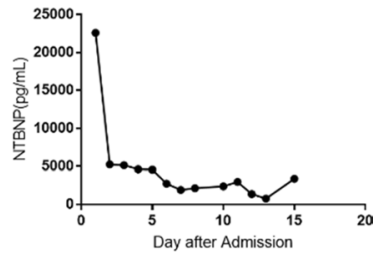

C

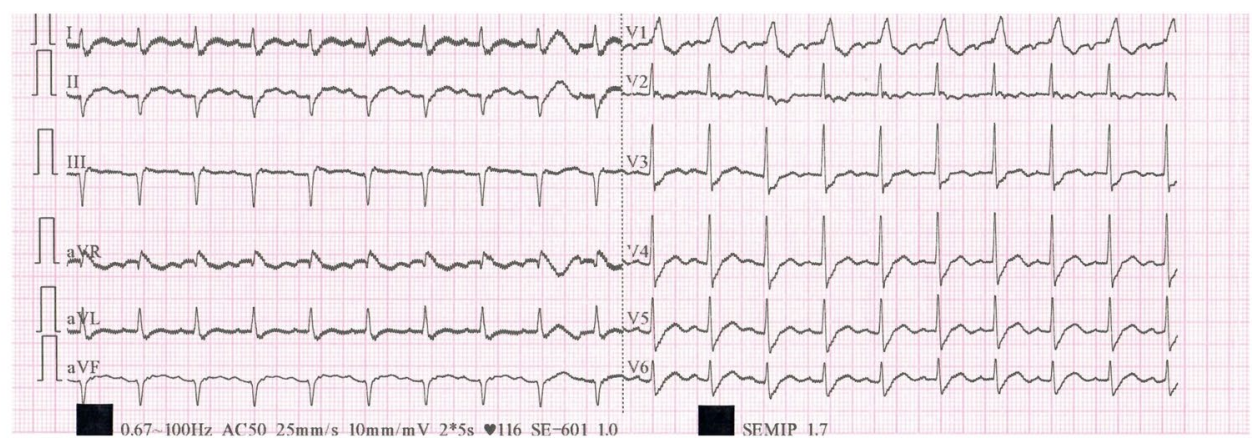

lesion had decreased. However, infection-related markers increased gradually from the 26th day, and vasoactive drugs were needed to maintain blood pressure. Candida, human $\alpha$-herpesvirus, and $\beta$-herpesvirus were detected in BALF on the 29th day, and blood culture was positive for Bacteroides ovalus, indicating the aggravation of secondary infection. Pulmonary artery systolic pressure (PASP) and tricuspid annular plane systolic excursion (TAPSE) showed sudden declines on the 26th and 29th days, suggesting impaired right cardiac function. After consultation with the expert group, anti-infection treatment was strengthened, but the effect was not good. Septic shock and disseminated intravascular coagulation (DIC) gradually developed, and ecchymosis was widely observed in the skin. The patient died on the 33rd day of hospitalization.

\section{Discussion}

Recently published COVID-19 studies have shown that cardiac complications, including heart failure, arrhythmia and myocardial infarction, are not uncommon, and the proportion of people with increased Trop I in severe cases is higher than other cases [3, 4]. However, to date, there is no case report that clearly suggests that COVID-19 can cause fulminant myocarditis. Fulminant myocarditis is mainly caused by a variety of viral infections and is characterized by the rapid occurrence of cardiogenic shock. In this case, the patient had the following features: sudden onset, obvious symptoms of viral infection, rapid emergence of severe hemodynamic disorders, severe myocardial injury, and diffuse decreased ventricular wall movement. The above features are all in accordance with the diagnostic criteria of fulminant myocarditis [5]. This patient's PASP gradually increased, suggesting that the pulmonary lesions were gradually aggravated. PASP and TAPSE suddenly decreased several days before death; this was considered to be related to functional decline in the right heart caused by sustained overload.

Clinical observation of 419 cases of COVID-19 in this hospital revealed that $32(8 \%)$ cases presented with elevated Trop I, including 8 (17\%) cases of severe pneumonia and 3 (21\%) cases of critical pneumonia. Echocardiography was performed in patients with abnormal myocardial injury markers, and only two patients were found to have reduced LVEFs and were diagnosed with fulminant myocarditis. Studies have confirmed that coronavirus infection can cause myocarditis and even congestive heart failure [6]. Other respiratory viruses have also been found to cause heart damage and coronary heart disease [7], and in this study, more than half of the patients who died had elevated Trop I during hospitalization. It has been reported that some severe acute respiratory syndrome (SARS) and Middle Eastern respiratory syndrome (MERS) patients have temporarily abnormal myocardial enzymes and may develop acute myocarditis and acute-onset heart failure $[8,9]$. In this case, the patient had no history of heart disease; therefore, the decreased cardiac function was likely to be caused by COVID-19.

Pathological changes in heart tissue may be directly due to virus replication in the myocardium or indirectly due to systemic responses to respiratory failure or a harmful immune responses caused by viral infection. In COVID19 autopsy studies, mononuclear inflammatory infiltration in the heart tissue, but no clear viral inclusion body was observed [10]. The rapid recovery of cardiac structure 


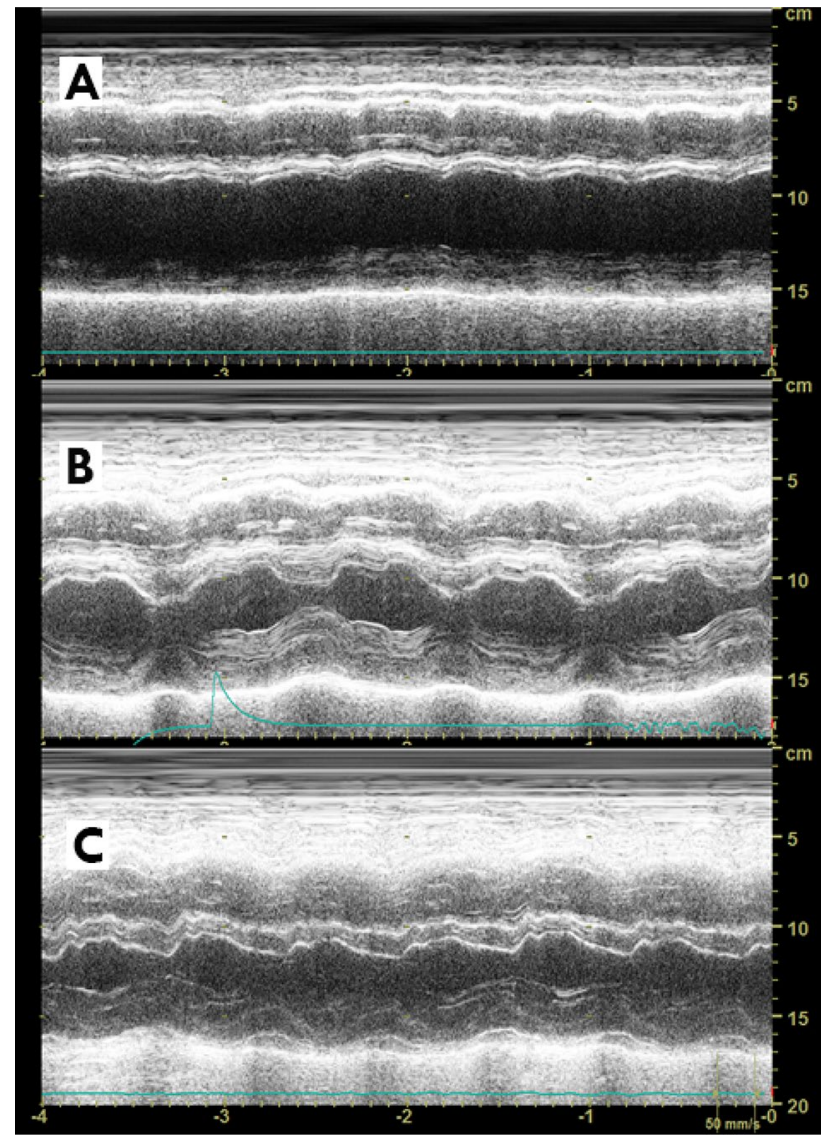

Fig. 4 The echocardiographic left ventricular M-mode images on the first day, 10th day, and 17th day after admission. a The left ventricular diameter was enlarged, and the ejection fraction was decreased on first day of admission; $\mathbf{b}$ this figure shows edema of left ventricular wall and improvement of LVEF. $\mathbf{c}$ The last bedside echocardiography examination showing a normal LVEF and wall thickness. $L V E F$ left ventricular ejection fraction

and function without significant decrease or even slight increase in viral load suggests that apart from viral replication in the myocardium, it is possible that immune response/cytokine storms may also play a significant role $[11,12]$. The patient in this case had significantly elevated IL-6, suggesting the presence of a cytokine storms. Cytokine storms may lead to increased vascular wall permeability and myocardial edema, which may explain the thickening of the interventricular septum in this patient. In addition, hypoxia can lead to early and substantial inflammatory responses and cell damage, which may cause rapid and considerable damage to the heart [13]. CRRT and ECMO remove some of the cytokines from the blood and increase blood oxygen saturation, reducing the excessive immune response of cytokines in the body and further reducing myocardial damage. Furthermore, a study showed that complete atrioventricular block, ventricular tachycardia, and fibrillation are risk factors for in-hospital death in fulminant myocarditis patients [14]. The absence of such electrocardiogram anomalies in this case may have contributed to the rapid improvement in the patient's heart condition, and the prognosis of this patient may have been better if secondary infection could have been controlled.

\section{Conclusion}

COVID-19 patients may develop severe cardiac complications such as myocarditis and heart failure. This is the first report of COVID-19 complicated with fulminant myocarditis. The lessons learned from this case are as follows: first, the heart is a secondary target organ after the lungs. It cannot only present with abnormalities in markers of myocardial injury, but also structural and functional damage. Second, immune-related injury may be the main mechanism of myocardial injury associated with viral infection. Third, the sudden decline in PASP and TAPSE may indicate that the course of disease is entering the late stage. The important question of whether COVID-19 can cause direct cardiac pathology requires further study.

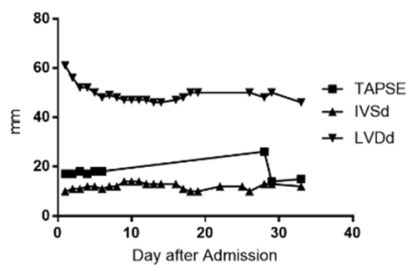

A

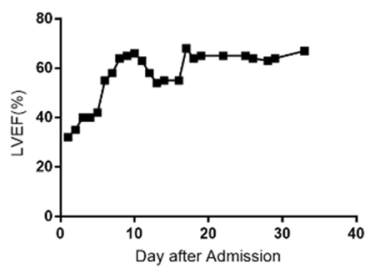

B

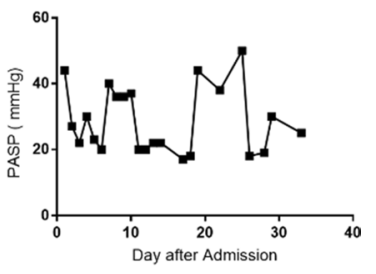

C
Fig. 5 Line chart of the echocardiography measurements. a The patients showed thickened IVSd and enlarged LVDd during treatment. TAPSE showed a sudden decline on the 29th day, suggesting impaired right cardiac function. The IVSd gradually thickened to a maximum thickness of $14 \mathrm{~mm}$. b A LVEF decrease appeared early during hospitalization and gradually returned to normal after treat- ment. c The PASP gradually increased as the disease progressed; however, a sudden drop in pulmonary artery pressure occurred on the 26th day. $L V D d$ left ventricular end-diastolic diameter, IVSd interventricular septum dimension, TAPSE tricuspid annular plane systolic excursion, $L V E F$ left ventricular ejection fraction, $P A S P$ pulmonary artery systolic pressure 


\section{Availability of data and material}

All available information is contained within the manuscript.

Acknowledgements We thank the patient for granting us permission to publish this information.

Author contributions JZ and CF had full access to all of the data of this case and take responsibility for the integrity and accuracy of the data. Concept and design: LL, CF, YW, and C-FD. Acquisition, analysis, or interpretation of data: all authors. Drafting of the manuscript: JZ. Critical revision of the manuscript for important intellectual content: JY, F-XW, W-BW, HG, and Y-XL. Supervision: LL, Y-XL, and CF.

Funding This manuscript was not funded.

\section{Compliance with ethical standards}

Conflict of interest On behalf of all authors, the corresponding author states that there are no conflicts of interest.

Ethics approval and consent to participate The study was approved by the Ethics Committee of Shenzhen Third People's Hospital on 11th Feb 2020 (No. 2020-006). Written informed consent was obtained from the patient.

Consent for publication Written informed consent was obtained from the patient for publication of this case and any accompanying images. A copy of the written consent is available for review by the Editor of this journal.

\section{References}

1. Huang C, Wang Y, Li X, Ren L, Zhao J, Hu Y, et al. Clinical features of patients infected with 2019 novel coronavirus in Wuhan, China. Lancet. 2020. https://doi.org/10.1016/S0140 $-6736(20) 30183-5$.

2. Li Q, Guan X, Wu P, Wang X, Zhou L, Tong Y, et al. Early transmission dynamics in Wuhan, China, of novel coronavirus-infected pneumonia. N Engl J Med. 2020. https://doi.org/10.1056/NEJMo a2001316.

3. Zhou F, Yu T, Du R, Fan G, Liu Y, Liu Z, et al. Clinical course and risk factors for mortality of adult inpatients with COVID-19 in Wuhan, China: a retrospective cohort study. Lancet. 2020. https ://doi.org/10.1016/S0140-6736(20)30566-3.

4. Wang D, Hu B, Hu C, Zhu F, Liu X, Zhang J, et al. Clinical characteristics of 138 hospitalized patients with 2019 novel coronavirus-infected pneumonia in Wuhan, China. JAMA. 2020. https:// doi.org/10.1001/jama.2020.1585.

5. Wang D, Li S, Jiang J, Yan J, Zhao C, Wang Y, et al. Chinese society of cardiology expert consensus statement on the diagnosis and treatment of adult fulminant myocarditis. Sci China Life Sci. 2019;62(2):187-202. https://doi.org/10.1007/s11427-018-9385-3.

6. Edwards S, Small JD, Geratz JD, Alexander LK, Baric RS. An experimental model for myocarditis and congestive heart failure after rabbit coronavirus infection. J Infect Dis. 1992;165(1):13440. https://doi.org/10.1093/infdis/165.1.134.

7. Blackburn R, Zhao H, Pebody R, Hayward A, Warren-Gash C. Laboratory-confirmed respiratory infections as predictors of hospital admission for myocardial infarction and stroke: timeseries analysis of English data for 2004-2015. Clin Infect Dis. 2018;67(1):8-17. https://doi.org/10.1093/cid/cix1144.

8. Han Y, Geng H, Feng W, Tang X, Ou A, Lao Y, et al. A followup study of 69 discharged SARS patients. J Tradit Chin Med. 2003;23(3):214-7.

9. Alhogbani T. Acute myocarditis associated with novel Middle east respiratory syndrome coronavirus. Ann Saudi Med. 2016;36(1):78-80. https://doi.org/10.5144/0256-4947.2016.78.

10. Xu Z, Shi L, Wang Y, Zhang J, Huang L, Zhang C, et al. Pathological findings of COVID-19 associated with acute respiratory distress syndrome. Lancet Respir Med. 2020. https://doi.org/10.1016/ S2213-2600(20)30076-X.

11. Channappanavar R, Perlman S. Pathogenic human coronavirus infections: causes and consequences of cytokine storm and immunopathology. Semin Immunopathol. 2017;39(5):529-39. https:// doi.org/10.1007/s00281-017-0629-x.

12. Huang KJ, Su IJ, Theron M, Wu YC, Lai SK, Liu CC, et al. An interferon-gamma-related cytokine storm in SARS patients. J Med Virol. 2005;75(2):185-94. https://doi.org/10.1002/jmv.20255.

13. Wu J, Stefaniak J, Hafner C, Schramel JP, Kaun C, Wojta J, et al. Intermittent hypoxia causes inflammation and injury to human adult cardiac myocytes. Anesth Analg. 2016;122(2):373-80. https ://doi.org/10.1213/ANE.0000000000001048.

14. Sawamura A, Okumura T, Ito M, Ozaki Y, Ohte N, Amano T, et al. Prognostic value of electrocardiography in patients with fulminant myocarditis supported by percutaneous venoarterial extracorporeal membrane oxygenation - analysis from the CHANGE PUMP study. Circ J. 2018;82(8):2089-95. https://doi.org/10.1253/ circj.CJ-18-0136. 